\title{
Leishmanicidal Activity of Oleuropein: Leishmania donovani Promastigote Cell Death through a Possibly ROS-Independent Mechanism
}

\author{
ID Kyriazis ${ }^{1,2}$, D Smirlis $^{3}$, A Papadaki $^{3}$, O Koutsoni ${ }^{2}$, N Aligiannis $^{4}$, AL Skaltsounis $^{4}$ and E Dotsika $^{2^{*}}$ \\ ${ }^{1}$ Department of Pharmacy, University of Patras, Rio, 26 500, Patra, Greece \\ ${ }^{2}$ Laboratory of Cellular Immunology, Hellenic Pasteur Institute, 127 Vasilissis Sofias Avenue, 115 21, Athens, Greece \\ ${ }^{3}$ Laboratory of Molecular Parasitology Hellenic Pasteur Institute, 127 Vasilissis Sofias Avenue, 115 21, Athens, Greece \\ ${ }^{4}$ Department of Pharmacognosy and Natural Products Chemistry, Faculty of Pharmacy, University of Athens, Panepistimiopolis Zografou, 15771 , Athens, Greece
}

*Corresponding author: Dotsika E, Laboratory of Cellular Immunology, Hellenic Pasteur Institute, 127 Vasilissis Sofias Avenue, 11521 , Athens, Greece, Tel: +302106478828; E-mail: e.dotsika@pasteur.gr

Received date: Sep 04, 2017; Accepted date: Sep 25, 2017; Published date: Sep 27, 2017

Copyright: ( 2017 Kyriazis ID, et al. This is an open-access article distributed under the terms of the Creative Commons Attribution License, which permits unrestricted use, distribution, and reproduction in any medium, provided the original author and source are credited.

\section{Abstract}

The development of potent and inexpensive antiparasitic agents for common use is imperative due to the absence of an effective and safe treatment for visceral leishmaniasis, a devastating parasitic disease. Oleuropein, a secoiridoid, exerts an antileishmanial effect on promastigotes of Leishmania infantum, L. major and L. donovani, as well as on amastigotes of $L$. donovani in in vitro and in vivo experimental models. In this study, our aim was to characterize how oleuropein drives parasites into cell death. Our hypothesis is that oleuropein promotes an apoptotis-like cell death which restrains the inflammatory processes that facilitate parasitic dissemination.
\end{abstract}

Logarithmic-phase $L$. donovani promastigotes were treated with oleuropein for 24,48 and $72 \mathrm{~h}$, and parasitic cell cycle, parasitic membrane asymmetry and intracellular generalized oxidative stress were assessed via flow cytometry. Morphological alterations were analyzed with confocal microscopy.

Cell-cycle analysis revealed that oleuropein fragmented parasite DNA within the first $24 \mathrm{~h}$ of incubation. In vitro treatment of promastigotes with oleuropein resulted in several morphological alterations and significant annexin $\mathrm{V}$ binding. Interestingly, oleuropein treatment did not increase the levels of intracellular reactive oxygen species until $48 \mathrm{~h}$ of incubation.

Our findings suggest that oleuropein promotes apoptosis-like cell death in $L$. donovani promastigotes that is not being mediated by an induced endoparasitic oxidative stress.

Keywords: Oleuropein, Leishmania donovani, Apoptosis, Leishmaniasis

\section{Abbreviations:}

$\mathrm{Ca}^{+2}$ : Calcium Ion; CM- $\mathrm{H}_{2}$ DCFDA: 5-(and-6)-chloromethyl-2',7'dichlorodihydrofluorescein diacetate, acetyl ester; FITC: Fluorescein Isothiocyanate; gMFI: Geometric Mean Fluorescence Intensity; PI: Propidium Iodide; ROS: Reactive Oxygen Species.

\section{Introduction}

Nature is the major source of novel chemical compounds with potential antiparasitic activity [1]. In particular, the olive tree, Olea europaea L. (Oleaceae), is of great interest because it contains chemical compounds with various biological activities. Oleuropein, the main phenolic compound in of $O$. europaea, is a secoiridoid glucoside (Figure 1) [2]. It is able to scavenge free radicals in order to protect cells from oxidation, leading to anti-aging and cardioprotective effects; it has also shown antitumor, antiviral, and antibacterial activities [2,3]. Previously, we demonstrated that oleuropein exerts in vitro antileishmanial activity on promastigotes and amastigotes of the genus Leishmania [4]. Moreover, we have showed that intraperitoneal administration of oleuropein diminishes the parasite burden in the visceral tissues in vivo, an effect that was maintained even 6 weeks after treatment [4]. The antiparasitic effect of oleuropein merits further investigation because its mechanism(s) of its action remains unknown. It is important to determine whether oleuropein causes leishmanicidal or leishmaniostatic effects and to determine the type of cell death that is initiated in Leishmania parasites.

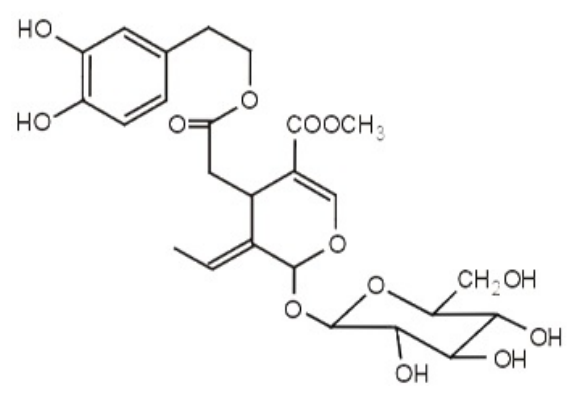

Figure 1: Molecular formula of oleuropein. 
Citation: Kyriazis ID, Smirlis D, Papadaki A, Koutsoni O, Aligiannis N, et al. (2017) Leishmanicidal Activity of Oleuropein: Leishmania donovani Promastigote Cell Death through a Possibly ROS-Independent Mechanism. J Pharmacogn Nat Prod 3: 141. doi: 10.4172/2472-0992.1000141

Page 2 of 7

The Nomenclature Committee on Cell Death put forward a list of phenotypical manifestations that define cell death [5], enabling us to use the term "apoptosis" when the cell death induced in parasites is circumscribed by these hallmarks. Oleuropein induces apoptosis in human skin carcinoma A-431 cells, a process that involves the activation of caspase-3 [20], and it inhibits cancer-cell proliferation by arresting the cell cycle phase in G0/G1 phase [6,7]. Moreover, oleuropein drives human colon adenocarcinoma HT-29 cells into a p53-dependent apoptosis [8]. Although Leishmania parasites do not contain any canonical regulators of apoptosis, such as the caspases and p53 [9], it is interesting to examine the mechanism by which oleuropein inhibits parasite growth. Parasite apoptosis is orchestrated mainly by a "biochemical triangle" that includes calcium ion $\left(\mathrm{Ca}^{+2}\right)$ levels, mitochondrial membrane potential and the production of intracellular reactive oxygen species (ROS). ROS production could be the stimulus that promotes alterations to $\mathrm{Ca}^{+2}$ homeostasis or the loss of physiological mitochondrial membrane potential, but it could also be the outcome of these biochemical activities [10].

Parasites of the genus Leishmania cause leishmaniasis, an inflammatory disease with a wide spectrum of clinical manifestations. Leishmaniasis is characterized by the WHO as a neglected disease; nearly 2 million new cases are diagnosed every year, leishmaniasis is endemic in 98 countries on five continents and the disease mostly affects "the poorest of the poor" [11]. Antileishmanial drugs such as pentavalent antimonials, paromomycin sulfate, miltefosine, and liposomal amphotericin B are associated with numerous drawbacks that extend from restricted activity and development of drug resistance to systemic toxicity and an intolerable cost for impoverished sufferers [12]. Due to these problems, in recent years there has been more interest in the study of ethnomedicines as a source of new chemotherapeutic compounds capable of eliminating parasites in the infected host with minimal side effects. Several natural products have been tested for antiparasitic activity and for the promotion of direct apoptosis-like death in Leishmania parasites [13].

During the initial establishment of parasitic infection, a subpopulation of Leishmania protozoans becomes altruistically apoptotic [14], enabling the rest of the population to infect host cells without activating defense mechanisms via inflammatory molecules. In contrast, after the onset of Leishmania infection, a non-inflammatory environment is not strategically correct for Leishmania parasites because tissue inflammation favors their spread. For that reason, a potent chemotherapeutic compound should promote apoptosis-like death of Leishmania in order to allow the host to utilize dynamic cellular immune responses to exterminate invasive parasites $[15,16]$ Our hypothesis is that oleuropein promotes an apoptotic-like cell death since it is already demonstrated in our previous research that oleuropein is able to promote a selective anti-inflammatory and antioxidant gene regulation in the environment of $L$. donovani-infected $\mathrm{BALB} / \mathrm{c}$ mice that is responsible for the induction of the desired Th1 immune response [17]. The aim of the present study was to shed light on biochemical and morphological alterations in Leishmania donovani Laveran and Laveran et Mesnil, 1903, Ross (Trypanosomatidae) promastigotes treated with oleuropein.

\section{Materials and Methods}

\section{Parasites}

The $L$. donovani reference strain (zymodeme MON-2, strain MHOM/IN/1996/THAK35) was used in this study. Promastigotes were cultured in complete medium consisting of RPMI-1640 (low phenol red content; Biochrom AG, Berlin, Germany) supplemented with 2 $\mathrm{mM}$ L-glutamine, $10 \mathrm{mM}$ HEPES, $24 \mathrm{mM} \mathrm{NaHCO}, 100 \mathrm{U} / \mathrm{ml}$ penicillin, $100 \mu \mathrm{g} / \mathrm{ml}$ streptomycin, and $10 \%(\mathrm{v} / \mathrm{v})$ heat-inactivated fetal bovine serum (Gibco, Paisley, UK) at $26^{\circ} \mathrm{C}$. Cultures were evaluated daily for parasite growth and promastigotes were collected at the middle of the logarithmic phase or at the beginning of the stationary phase of growth.

\section{Oleuropein}

Oleuropein was obtained from olive leaves ( $O$. europeae var. microcarpa alba or koroneiki) as described elsewhere [4]. Purity was determined using high-performance liquid chromatography (HPLC) and nuclear magnetic resonance (NMR) spectroscopy (Figure 2). Quantitative determination of oleuropein purity was performed with a Thermo Finnigan HPLC system (Thermo Electron Corporation, San Jose, California, San Jose, CA, USA) coupled with a Spectra System UV6000LP PDA detector (Thermo Electron Corporation). A twosolvent gradient method was used: solvent $\mathrm{A}, \mathrm{H}_{2} \mathrm{O}+1 \%$ acetic acid $(\mathrm{v} / \mathrm{v})$; solvent $\mathrm{B}$, methanol. The flow rate was $1 \mathrm{ml} / \mathrm{min}$ and the following elution program was applied: 0-2 min, linear gradient to $5 \%$ solvent B; 2-10 min, linear gradient to $25 \%$ solvent $\mathrm{B} ; 10-20 \mathrm{~min}$, linear gradient to $40 \%$ solvent B; $20-30 \mathrm{~min}$, linear gradient to $50 \%$ solvent $\mathrm{B}$; 30-34 min, $50 \%$ solvent B, isocratic; $34-45$ min, linear gradient to $90 \%$ solvent $\mathrm{B}$; 45-50 min, 90\% solvent $\mathrm{B}$, isocratic; 50-60 min, linear gradient to $100 \%$ solvent $\mathrm{B}$; $60-65 \mathrm{~min}, 100 \%$ solvent $\mathrm{B}$, isocratic. A standard solution of oleuropein was prepared in 50:50 $\mathrm{H}_{2} \mathrm{O}$ /methanol. Separation was performed at $25^{\circ} \mathrm{C}$ and the injection volume was $20 \mu \mathrm{l}$. Detection was performed at $248 \mathrm{~nm}$ with a Supelco Analytical Discovery HS C18 column (Sigma-Aldrich, Taufkirchen, Germany) $(25 \mathrm{~cm}, 4.6 \mathrm{~mm}$, inside diameter $5.0 \mu \mathrm{m})$. O. europea var. koroniki is one of the most popular cultivates in Greece; and its leaves are a rich source of oleuropein, which and its percentage can constitute nearly $10 \%$ of dry weight. Xynos et al. Oleuropein was diluted in distilled water before use, filtered with a $0.45 \mu \mathrm{m}$ filter (Millex; Merck, Billerica, MA, USA), and stored at $4^{\circ}$ C. Logarithmic-phase $L$. donovani promastigotes were exposed to two concentrations of oleuropein: $128.4 \mu \mathrm{M}(69.4 \mu \mathrm{g} / \mathrm{ml})$, which is the half- maximal inhibitory concentration, and $256.8 \mu \mathrm{M}(138.8 \mu \mathrm{g} / \mathrm{ml})$, which is twice the half-maximal inhibitory concentration [4]. Higher concentrations were deemed impractical and without scientific importance because similar concentrations previously displayed a cytotoxic effect (CC50) against J774A.1 macrophages [4] 
Citation: Kyriazis ID, Smirlis D, Papadaki A, Koutsoni O, Aligiannis N, et al. (2017) Leishmanicidal Activity of Oleuropein: Leishmania donovani Promastigote Cell Death through a Possibly ROS-Independent Mechanism. J Pharmacogn Nat Prod 3: 141. doi: $10.4172 / 2472-0992.1000141$

Page 3 of 7

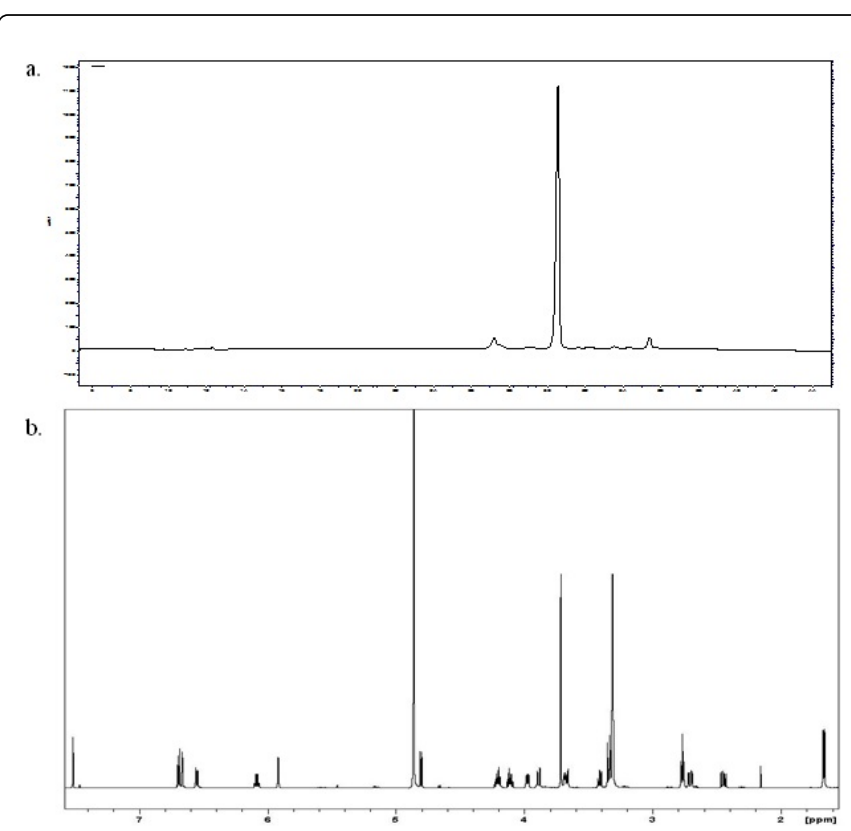

Figure 2: Oleuropein purity was $>95 \%$ according to (A) HPLC (RP-18) chromatogram $(240 \mathrm{~nm})$ and (B) nuclear magnetic resonance spectroscopy spectrum $(\mathrm{CD} 3 \mathrm{OD}, 600 \mathrm{MHz})$.

\section{Promastigote cell-cycle analysis}

We used flow cytometry to evaluate the effects of oleuropein on the L. donovani promastigote cell cycle. Oleuropein $(128.4 \mu \mathrm{M}$ and 256.8 $\mu \mathrm{M})$ was added to cultures of $2 \times 10^{6}$ parasites $/ \mathrm{ml}$ of $L$. donovani promastigotes in logarithmic growth and the cultures were incubated at $26^{\circ} \mathrm{C}$. The negative control consisted of similar parasite cultures without oleuropein. After $24 \mathrm{~h}, 48 \mathrm{~h}$, and $72 \mathrm{~h}$ of incubation, $1 \mathrm{ml}$ was removed from each culture and the parasites were counted. Parasites $\left(10^{6}\right)$ from each group were incubated with $100 \%$ ethanol (v/v) for 2 min and washed with phosphate-buffered saline supplemented with $100 \mu \mathrm{g} / \mathrm{ml}$ RNAse A (Promega, Madison, WI, USA) and $50 \mu \mathrm{g} / \mathrm{ml}$ propidium iodide (PI). Cells were incubated for another $1 \mathrm{~min}$ at room temperature and placed at $4^{\circ} \mathrm{C}$ until use. Flow cytometry (FACS Calibur; Becton-Dickinson, San Jose, CA, USA) was used to evaluate 10,000 promastigotes per experimental group and the results were plotted with Cell Quest Pro Version 5.2.1. (Becton Dickinson) and Flowjo V.10.0.8 (TreeStar Inc., Ashland, OR, USA).

\section{Staining with annexin V-fluorescein isothiocyanate (FITC)}

In order to evaluate the effect of oleuropein on $L$. donovani membrane integrity, we used annexin V-FITC and PI from the Apoptosis Detection Kit I (Becton-Dickinson) in conjunction with flow cytometry. Briefly, $128.4 \mu \mathrm{M}$ and $256.8 \mu \mathrm{M}$ oleuropein were added to fresh parasite cultures (starting inoculum of $2 \times 10^{6} \mathrm{~L}$. donovani promastigotes $/ \mathrm{ml}$ in mid-logarithmic growth), which were incubated at $26^{\circ} \mathrm{C}$ in $25^{-\mathrm{cm} 2}$ culture flasks. The negative control lacked oleuropein. After $24 \mathrm{~h}, 48 \mathrm{~h}$, and $72 \mathrm{~h}$ of incubation, $1 \mathrm{ml}$ was removed from each culture and parasites were counted. As a positive for inducing necrosis, we added Triton X-100 $(0.1 \%, \mathrm{v} / \mathrm{v})$ to the culture medium for $5 \mathrm{~min}$ to perforate the cell membranes of the promastigotes. Subsequently, $10^{6}$ promastigotes from each group (negative control, positive control,
128.4 $\mu \mathrm{M}$ oleuropein, and $256.8 \mu \mathrm{M}$ oleuropein) were washed with icecold phosphate-buffered saline and then with annexin binding buffer. One hundred microliters $\left(10^{5}\right.$ promastigotes) from each group were placed in separate eppendorf tubes and were incubated for $15 \mathrm{~min}$ at $26^{\circ} \mathrm{C}$ with $10 \mu \mathrm{l}$ annexin V-FITC and $10 \mu \mathrm{l} \mathrm{PI}$ in accordance with the manufacturer's instructions. Samples were placed at $4^{\circ} \mathrm{C}$ for flow cytometry (10,000 promastigotes per experimental group). Cells that were not stained with both annexin V-FITC and PI were considered healthy. Cells that were stained with annexin V-FITC but not with PI were considered to be in early apoptosis. Cells that were stained with both annexin V-FITC and PI were alive or already dead and considered to be in late apoptosis or in necrosis respectively.

\section{Confocal microscopy}

Oleuropein $(256.8 \mu \mathrm{M})$ was added to a culture with $2 \times 10^{6}$ parasites/ml ( $L$. donovani promastigotes in logarithmic growth) at $26^{\circ} \mathrm{C}$ for $24 \mathrm{~h}$. The negative control consisted of a similar culture without oleuropein. After incubation, the parasites were washed in phosphate-buffered saline and resuspended in paraformaldehyde $2 \%$ $(\mathrm{v} / \mathrm{v})$ for $15 \mathrm{~min}$ at room temperature. Parasites were washed again with phosphate-buffered saline $(50 \mu \mathrm{l} / \mathrm{sample})$ to remove paraformaldehyde and placed on coverslips coated with $1 \mathrm{mg} / \mathrm{ml}$ polyL-lysine (Sigma-Aldrich). After an 18-h incubation at room temperature to permit the promastigotes to attach to the coverslips, the cells were incubated for $1 \mathrm{~min}$ in $0.1 \%$ Triton X-100 (v/v) to perforate cellular membranes. Parasites were incubated with $50 \mu \mathrm{g} / \mathrm{ml}$ RNase A for $1 \mathrm{~h}$ at room temperature, and then with $100 \mu \mathrm{g} / \mathrm{ml}$ PI for another hour under the same conditions. The coverslips were mounted with 10 $\mu \mathrm{L}$ Mowiol 4-88 (25\% v/v glycerol, $100 \mathrm{mM}$ Tris-HCl, $\mathrm{pH}$ 8.5; Calbiochem -Merck), on microscope slides, sealed with nail polish and stored at $4^{\circ} \mathrm{C}$. After $18 \mathrm{~h}$ at $4^{\circ} \mathrm{C}$, slides were subjected to confocal fluorescence microscopy (TCS-SP; Leica Microsystems, Wetzlar, Germany). At least 15 cells from three independent experiments were observed from each experimental group.

\section{ROS production in parasites}

We also used flow cytometry to assess the effect of oleuropein on intracellular ROS production in logarithmic phase $L$. donovani promastigotes. Oleuropein $(128.4 \mu \mathrm{M}$ and $256.8 \mu \mathrm{M})$ was added to cultures of $2 \times 10^{6}$ parasites $/ \mathrm{ml}$, and the cultures were incubated at $26^{\circ} \mathrm{C}$. The negative control lacked oleuropein. After $2 \mathrm{~h}, 4 \mathrm{~h}, 8 \mathrm{~h}, 12 \mathrm{~h}$, $18 \mathrm{~h}, 24 \mathrm{~h}, 48 \mathrm{~h}$, and $72 \mathrm{~h}$ incubation, $1 \mathrm{ml}$ was removed from each culture and the parasites were counted. Then, $10^{6}$ parasites from each group were incubated with $5 \mu \mathrm{M}$ 5-(and-6)-chloromethyl-2', $7^{\prime}$ dichlorodihydro-fluorescein diacetate, acetyl ester $\left(\mathrm{CM}-\mathrm{H}_{2} \mathrm{DCFDA}\right.$; Thermo Fisher Scientific, Waltham, MA, USA) for $45 \mathrm{~min}$ at $26^{\circ} \mathrm{C}$ in the dark. Positive-control cells were parasites from the negative-control culture that were incubated with $10 \mu \mathrm{M}$ hydrogen peroxide for another 30 min under the same conditions. Parasites from each experimental group were washed with phosphate-buffered saline and placed at $4^{\circ} \mathrm{C}$ until use. Flow cytometry was used to evaluate 10,000 promastigotes per experimental group.

\section{Statistical analysis}

Three independent experiments were carried out in duplicate for each analysis. The nonparametric Mann-Whitney test (SPSS; Statistical Package for Social Sciences, Version 14.0) was used to identify significant differences between in vitro experiments. $\mathrm{P}$ values $<0.05$ were considered significant. 
Citation: Kyriazis ID, Smirlis D, Papadaki A, Koutsoni O, Aligiannis N, et al. (2017) Leishmanicidal Activity of Oleuropein: Leishmania donovani Promastigote Cell Death through a Possibly ROS-Independent Mechanism. J Pharmacogn Nat Prod 3: 141. doi: $10.4172 / 2472-0992.1000141$

Page 4 of 7

\section{Results}

\section{Oleuropein causes DNA fragmentation in L. donovani promastigotes}

First, we investigated whether oleuropein influences cell-cycle progression and DNA integrity in $L$. donovani promastigotes (Figure 3). After only $24 \mathrm{~h}$ of incubation, both oleuropein concentrations caused a significant increase $(\mathrm{P}=0.05)$ in the number of parasites with incomplete DNA load (in the subG0 phase). At this time point, parasites in subG0 treated with $128.4 \mu \mathrm{M}$ and $256.8 \mu \mathrm{M}$ oleuropein constituted $26.4 \pm 3.5 \%$ and $35.7 \pm 3.7 \%$ of the population, respectively, while only $8.4 \pm 5 \%$ of negative-control parasites were in subG0 (Figure 3). At $48 \mathrm{~h}$ and $72 \mathrm{~h}$, the percentage of parasites in subG0 in each experimental group was lower than at $24 \mathrm{~h}$, but significantly more parasites exposed to oleuropein were in subG0 than were negativecontrol parasites at these time points (Figure 3). This decrease was expected because the rest of the population continues to divide, thus decreasing the percentage of the population in subG0. Consistent with this observation, at $24 \mathrm{~h}$, the percentage of the parasite population in $\mathrm{G} 1$ was significantly lower $(\mathrm{P}=0.05)$ after exposure to $128.4 \mu \mathrm{M}(55.8 \pm$ $5.2 \%)$ and $256.8 \mu \mathrm{M}$ oleuropein $(47.6 \pm 0.4 \%)$ than after no exposure $(67.1 \pm 10.2 \%$; Figure 3). At $48 \mathrm{~h}$ and $72 \mathrm{~h}$, the percentages of G1-phase parasites exposed to the two oleuropein concentrations were significantly higher than the percentage of negative-control parasites in G1 (Figure 3). The few significant between-group differences in $S$ and G2-M phases did not suggest that either oleuropein concentration affected cell-cycle progression in $L$. donovani promastigotes.

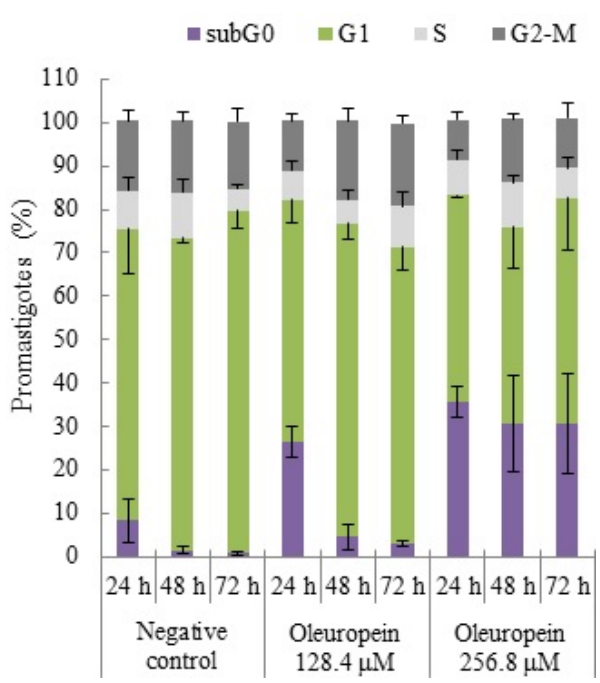

Figure 3: Effect of oleuropein on the cell cycle of $L$. donovani promastigotes. ${ }^{*} \mathrm{P}<0.05$ versus the negative control. ${ }^{\wedge} \mathrm{P}<0.05$ versus the other oleuropein concentration. Data represent the average \pm standard deviation of three independent experiments.

\section{The effect of oleuropein on parasite membrane asymmetry}

The DNA fragmentation detected in our cell-cycle analysis (Figures 3 and 4) suggests that oleuropein exerts leishmanicidal rather than leishmaniostatic effect. To confirm the presence of apoptosis-like cell death, we sought to identify membrane asymmetry and membrane impermeability via annexin V staining (Figure 5). At $24 \mathrm{~h}$, only $2 \pm$
$0.5 \%$ of negative-control parasites appeared to be annexin V+/PI(early apoptosis). Parasites exposed to the low dose of oleuropein exhibited a similar rate of annexin V+/PI- staining ( $4.7 \pm 4 \%)$, while exposure to the high concentration was associated with a significant increase $(9.3 \pm 5.5 \%, \mathrm{P}=0.05$; Figure 5$)$. At $24 \mathrm{~h}$, only $1.9 \pm 0.5 \%$ of negative-control cells were annexin $\mathrm{V}+\mathrm{PI}+$ (in late apoptosis or necrotic; Figure 5). Incubation with the low dose of oleuropein did not alter the percentage of annexin $\mathrm{V}+\mathrm{PI}+$ parasites, but incubation with $256.8 \mu \mathrm{M}$ oleuropein induced a significant 3 -fold increase $(6.1 \pm 2.4 \%$, $\mathrm{P}=0.05$ ) versus the other two experimental groups (Figure 5).

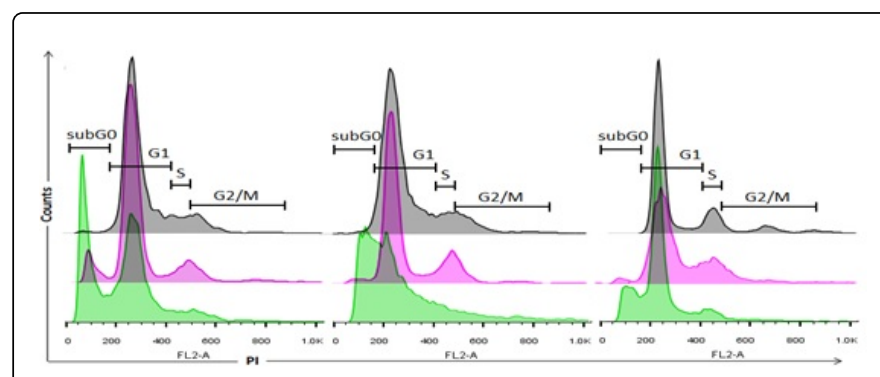

Figure 4: Semi-merged histograms of flow-cytometry data from oleuropein-treated L. donovani (left to right, $24 \mathrm{~h}, 48 \mathrm{~h}$, and $72 \mathrm{~h}$ of oleuropein exposure). Black, negative control (no exposure); purple, $128.4 \mu \mathrm{M}$ oleuropein; green, $256.8 \mu \mathrm{M}$. Data represent one of three independent experiments.

At $48 \mathrm{~h}$, the rates of negative-control parasites in early $(1.6 \pm 1.1 \%)$ and late apoptosis or necrosis $(1.3 \pm 0.9 \%)$ remained low (Figure 5); these percentages were higher in the presence of $128.4 \mu \mathrm{M}$ oleuropein ( $4.5 \pm 1.8 \%$ and $3.6 \pm 1.2 \%$, respectively, $\mathrm{P}=0.05$; Figure 5$)$. However, $256.8 \mu \mathrm{M}$ oleuropein significantly increased the percentage of annexin $\mathrm{V}+$ cells $(4.7 \pm 0.9 \%$ and $18.7 \pm 5.8 \%$, respectively, $\mathrm{P}=0.05$; Figure 5 ).

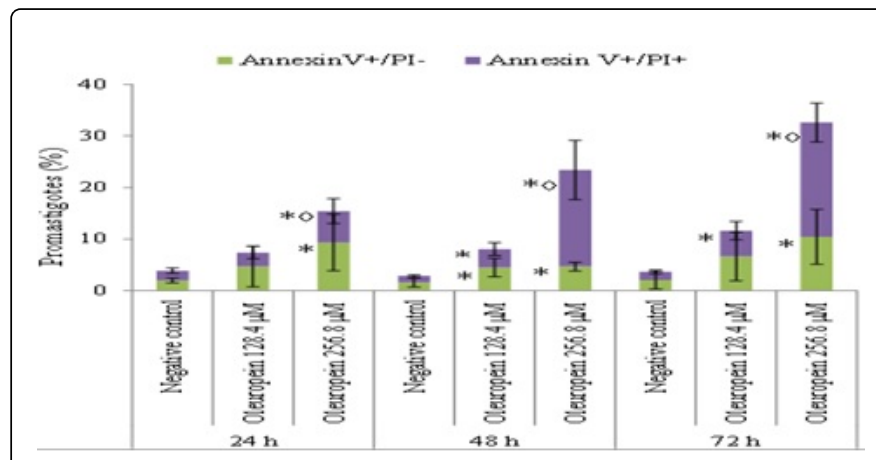

Figure 5: Effect of oleuropein on membrane asymmetry in $L$. donovani promastigotes, as assayed via double staining with annexin $\mathrm{V}$ and $\mathrm{PI} .{ }^{*} \mathrm{P}<0.05$ versus the negative control. ${ }^{\diamond} \mathrm{P}<0.05$ versus the other oleuropein dose. Data represent the average \pm standard deviation of three independent experiments.

At $72 \mathrm{~h}$, few parasites in the negative-control group were in early $(2.1 \pm 1.7 \%)$ or late apoptosis/necrosis $(1.6 \pm 0.4 \%$; Figure 5$)$. There was a three-fold increase in annexin $\mathrm{V}+$ staining after exposure to 128.4 $\mu \mathrm{M}$ oleuropein, but only the percentage of annexin $\mathrm{V}+/ \mathrm{PI}+$ cells $(5 \pm 1.8 \%)$ was significantly $(\mathrm{P}=0.05)$ higher than the respective percentage of parasites without exposure to oleuropein (Figure 5). In contrast, in parasites exposed to $256.8 \mu \mathrm{M}$ oleuropein, the percentages 
Citation: Kyriazis ID, Smirlis D, Papadaki A, Koutsoni O, Aligiannis N, et al. (2017) Leishmanicidal Activity of Oleuropein: Leishmania donovani Promastigote Cell Death through a Possibly ROS-Independent Mechanism. J Pharmacogn Nat Prod 3: 141. doi: $10.4172 / 2472-0992.1000141$

Page 5 of 7

of annexin $\mathrm{V}+/ \mathrm{PI}$ - cells $(10.5 \pm 5.3 \%)$ and annexin $\mathrm{V}+/ \mathrm{PI}+$ cells $(22.1 \pm$ $3.8 \%)$ significantly increased $(\mathrm{P}=0.05)$. The positive control, which consisted of promastigotes exposed to Triton X-100, contained $2.4 \pm$ $1.3 \%$ annexin V+/PI- cells and $44.4 \pm 3.5 \%$ annexin $\mathrm{V}$-/PI+ (necrotic) cells (Figure 6).

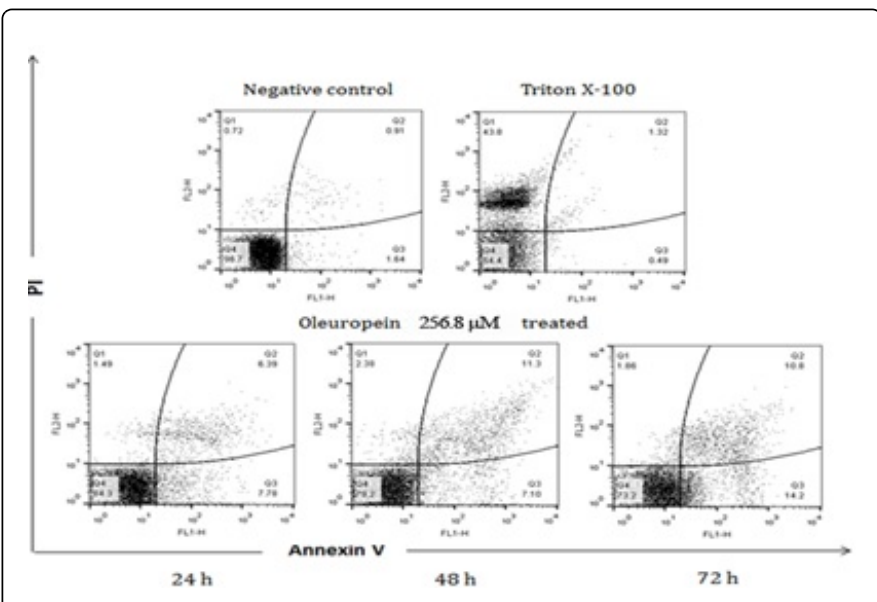

Figure 6: Dot plots of annexin V-FITC and PI double staining. Data represent one of three independent experiments.

\section{Morphological alterations in oleuropein-treated $L$. donovani promastigotes}

The biochemical markers examined above highlight the ability of $256.8 \mu \mathrm{M}$ oleuropein to promote apoptosis in Leishmania parasites after only $24 \mathrm{~h}$ of incubation. Using confocal microscopy and PI staining, we examined the morphological changes in the cells and nuclei of $L$. donovani promastigotes after $24 \mathrm{~h}$ of exposure to oleuropein (Figure 7).

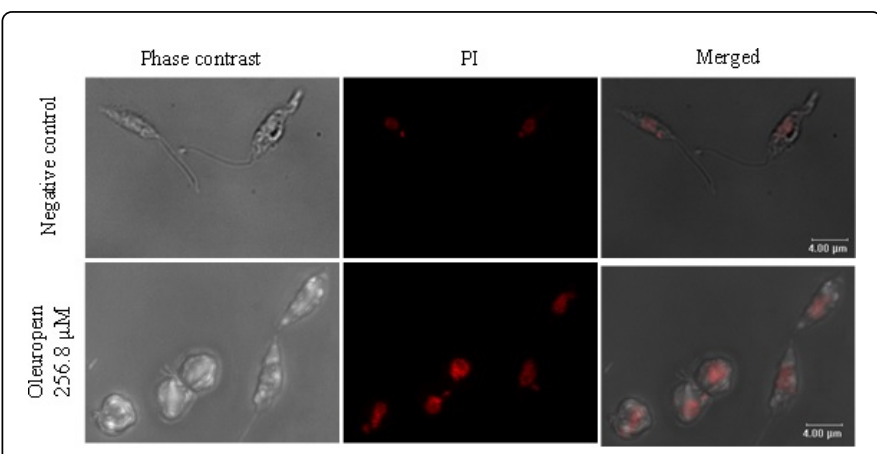

Figure 7: Cellular and nuclear morphology induced after exposure of $L$. donovani logarithmic phase promastigotes to oleuropein. Data represent one of three independent experiments.

In the absence of oleuropein, $L$. donovani promastigotes exhibited canonical morphological manifestations, including a typical elongated cell shape with a long flagellum and a spherical and well-stained nucleus and kinetoplast (Figure 7). In contrast, parasites treated with $256.8 \mu \mathrm{M}$ oleuropein for $24 \mathrm{~h}$ were round, had decreased cellular volume, and had lost a substantial portion of their flagella; in some cases, the flagellum was completely missing (Figure 7). After exposure to oleuropein, the fluorescence of apoptotic nuclei was more intense than the fluorescence of the nuclei of non-treated parasites (Figure 7). This intensity, which was also higher in the kinetoplast of oleuropeinexposed parasites, indicated chromatin condensation. These phenotypes demonstrate that oleuropein promotes apoptosis-like death in these parasites.

\section{Endoparasitic ROS Production in Oleuropein-Treated L. donovani Promastigotes}

Intracellular production of ROS may constitute the cause or the effect of apoptosis in normal eukaryotic parasites. The time course of ROS production in terms of other biochemical parameters is critical in determining whether the apoptotic mechanism involves ROS generation (Figure 8). We selected $\mathrm{CM}-\mathrm{H}_{2} \mathrm{DCFDA}$ as an indicator of general oxidative stress [18], and our flow-cytometry experiments evaluated all possible cell populations (live cells, apoptosis-like cells, and dead cells). ROS may not be detectable in dead protozoans [19].

At each timepoint, the ROS levels in the positive-control cultures significantly differed $(\mathrm{P}=0.05)$ from those of the other three experimental groups, with values ranging from $9.5 \pm 0.8$ geometric mean fluorescence intensity (gMFI) to $10.5 \pm 0.3$ gMFI (Figure 8). Apart from the positive-control we did not observe any increase in the level of endoparasitic ROS up to $48 \mathrm{~h}$ among the rest experimental groups (Figure 8 ); at $48 \mathrm{~h}$, only the high dose of oleuropein prompted a significant $(\mathrm{P}=0.05)$ change in ROS levels $(2.6 \pm 0.9 \mathrm{gMFI})$ versus the negative control $(0.6 \pm 0.2$ gMFI; Figure 8$)$. At $72 \mathrm{~h}$, both oleuropein concentrations caused significant $(\mathrm{P}=0.05)$ increases in ROS levels $(1.6$ \pm 0.6 gMFI and $7.3 \pm 0.1$ gMFI, respectively) versus the negative control (Figure 8).

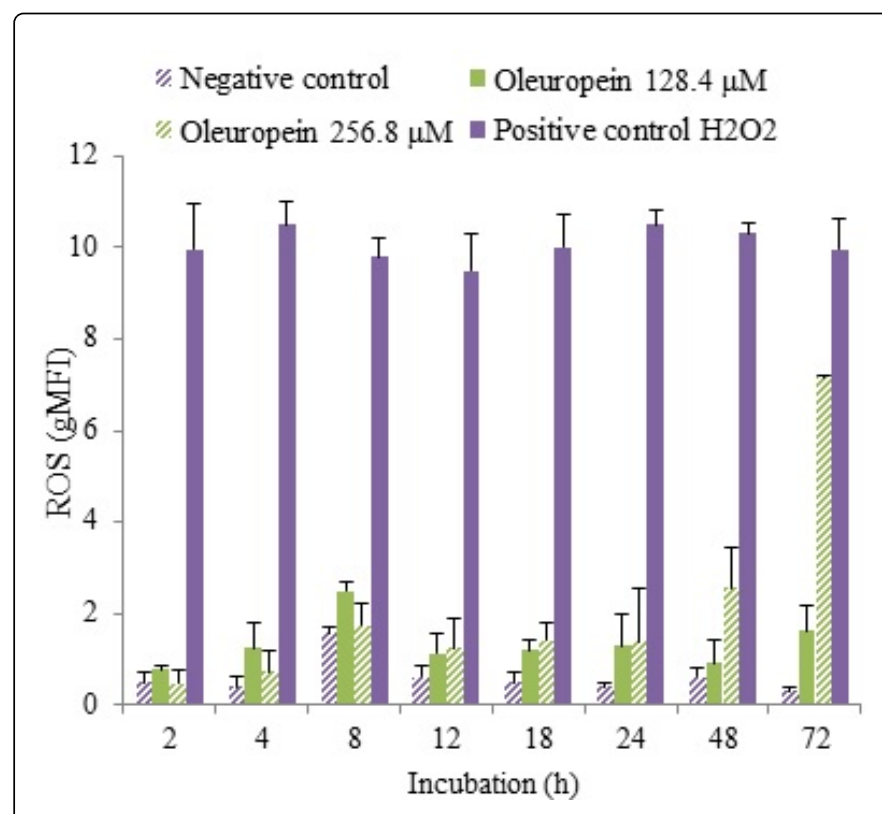

Figure 8: Effect of oleuropein on endoparasitic ROS levels. ${ }^{*} \mathrm{P}<0.05$ versus the negative control. Data represent the average \pm standard deviation of three independent experiments.

\section{Discussion}

The ability of oleuropein to promote apoptosis in various mammalian cell lines through caspases, p53, and Bcl-2 $[8,20]$ 
Citation: Kyriazis ID, Smirlis D, Papadaki A, Koutsoni O, Aligiannis N, et al. (2017) Leishmanicidal Activity of Oleuropein: Leishmania donovani Promastigote Cell Death through a Possibly ROS-Independent Mechanism. J Pharmacogn Nat Prod 3: 141. doi: 10.4172/2472-0992.1000141

Page 6 of 7

prompted our interest in the mechanism by which this biophenol drives L. donovani parasites into apoptosis. Parasites of the Leishmania genus lack these regulators of apoptosis, suggesting that protozoan apoptosis should be driven by different mechanisms $[9,10]$. The parasite transition to apoptosis is expected to be associated with a noninflammatory environment during parasite clearance in vivo, favoring the required immune response by type $1 \mathrm{~T}$ helper cells $[14,16,21]$. In a previous study, oleuropein reduced the proliferation of $L$. donovani promastigotes, as revealed with the AlamarBlue assay [4]. This colorimetric method detects decreases in the metabolism of cultured protozoans without providing mechanistic details. These decreases may be due to the leishmanicidal or leishmaniostatic action of the potential pharmaceutical agent.

Notably, we observed inconsistent results when we attempted to assay DNA fragmentation via gel electrophoresis and DNA staining with ethidium bromide (data not shown). We therefore chose to assess antileishmanial activity by evaluating the cell cycle using flow cytometry. Previously, this method was used to detect the leishmanicidal activity of pure constituents of Asparagus racemosus (Asparagaceae) (racemoside A) and Artemisia annua L. (Asteraceae), (artemisinin) [22,23]. These natural extracts induced genome fragmentation, which resulted in the production of parasites with incomplete DNA load. On the other hand, taxol, which is derived from Taxus baccata L. (Taxaceae), halts $L$. donovani mitosis at G2-M phase, thus exerting leishmaniostatic activity [24]. Oleuropein appears to exhibit leishmanicidal activity since it is able to increase the percentage of $L$. donovani promastigotes with incomplete DNA load after $24 \mathrm{~h}$ of incubation (Figures 3 and 4). Other flavonoids, such as luteolin and quercetin, display the same activity against Leishmania promastigotes, but the effect becomes pronounced after more than $24 \mathrm{~h}$ of exposure $[22,23,25]$. After $72 \mathrm{~h}$ of incubation, oleuropein, luteolin, and quercetin all lead to cell-cycle arrest in $30 \%$ of the promastigote culture (Figure 3) $[25]$.

The extent of $L$. donovani DNA fragmentation detected here is not sufficient to indicate that these cells undergo apoptosis (Figure 3). Additional phenotype and biochemical information is needed to characterize apoptosis in promastigotes, a process that is followed, to some extent, by eukaryotic parasites [9]. The basic phenotype of apoptosis in these parasites is membrane asymmetry; annexin $\mathrm{V}$ binding phospholipids such as phosphatidylinositol, ethanolamine, phosphatidylglycerol, and phosphatidic acid are redistributed in the plasma membrane [26,27]. Racemoside A, Aloe vera (L.) Burm. f. (Xanthorrhoeaceae) extract, and the methanolic extract of Piper betle L. (Piperaceae) exhibit different patterns of action over time [22,28]. Racemoside A induces plasma-membrane asymmetry after $30 \mathrm{~min}$ of incubation, and after $6 \mathrm{~h}$, the majority of parasites are annexin V-/PI+, reflecting necrosis. Aloe vera extract promotes the highest percentages of annexin V+ cells at $24 \mathrm{~h}$, whereas luteolin and quercetin cause the highest percentages of annexin $\mathrm{V}+$ cells after $48 \mathrm{~h}$ of incubation, due to delayed genome fragmentation [25]. In the present study, oleuropein exposure led to phenotypes that were similar to those induced by Aloe vera extract, since the percentage of annexin $\mathrm{V}+$ parasites was significantly higher after only $24 \mathrm{~h}$ of incubation (Figure 5). However, at $48 \mathrm{~h}$ and $72 \mathrm{~h}$, it seemed that cells moved from an early apoptotic state (annexin V+/PI-) to a late-apoptotic or necrotic state (Annexin V $+/ \mathrm{PI}+$ ), after which the population underwent a new parasitic entry into the early apoptotic stage (Figure 6).

Here, DNA fragmentation and membrane asymmetry, the classic hallmarks of apoptosis, were confirmed via confocal microscopy. The cellular and nuclear morphologies of $L$. donovani were altered after a 24-h incubation with a high dose of oleuropein (Figure 7). Exposed parasites were rounded, had lost part of their flagella, had diminished cellular volume, and harbored condensed chromatin. Previously, racemoside A, taxol, luteolin, and quercetin caused similar cellular damage, abrogating cellular cohesion and disrupting the architecture of the parasite's nucleus $[22,24,25]$. In contrast, exposure to Aloe vera extract previously led to chromatin condensation only (Dutta et al., 2007a), and 10-deacetylbaccatin III, a taxol derivative, did not alter parasite morphology $[19,24]$.

The disturbance of cellular physiology in the parasite is orchestrated by a "biochemical triangle" that includes endoparasitic ROS levels, Ca +2 homoeostasis, and mitochondrial membrane potential [10]. Numerous stimuli have the ability to raise endoparasitic $\mathrm{Ca}^{+2}$ levels, a phenomenon that promotes the disruption of mitochondrial membrane potential. This biochemical cascade is followed by ROS production due to oxidative phosphorylation [29]. Other stimuli, such as camptothecin, curcumin, thenoyltrifluoro-acetone, and hydrogen peroxide, increase endoparasitic ROS levels and lead to lipid peroxidation [30]. This process affects membrane asymmetry and $\mathrm{Ca}^{+2}$ channels and is followed by a loss of mitochondrial membrane potential. On the other hand, antimony potassium tartrate promotes ROS production, which first disrupts mitochondrial membrane potential and then increases $\mathrm{Ca}^{+2}$ levels [31]. Aloe vera extract employs a different mechanism of action; the disruption of mitochondrial membrane potential is not followed by increases in ROS and/or $\mathrm{Ca}^{+2}$ [19]. The mechanism of oleuropein seem to be similar to that of Aloe vera, since endoparasitic ROS levels were significantly higher after $48 \mathrm{~h}$ of exposure (Figure 8). After $48 \mathrm{~h}$, membrane asymmetry was advanced (Figure 5), a high number of parasites were in subG0 phase (Figure 3), and the parasites displayed morphological alterations (Figure 7). Our data suggest that ROS production derives from extensive damage to the parasite and does not activate mechanisms that lead these protozoans into apoptosis.

\section{Conclusions}

The data presented here constitute the first evidence that oleuropein promotes the apoptosis of $L$. donovani promastigotes, a process that may not interfere with ROS production. This outcome may contribute to the effective suppression of amastigote proliferation in the infected host, which in a previous in vivo visceral model resulted in a lower parasite burden in the spleen and liver [4]. Controlled parasite apoptosis likely does not trigger inflammatory responses within the parasite's niche, enabling the host's immune response to undergo type 1 $\mathrm{T}$ helper cell polarization and to activate the production of host microbicidal molecules such as ROS and reactive nitrate intermediates.

\section{Conflict of interest}

The authors declare no financial or commercial conflicts of interest.

\section{Acknowledgements}

We thank Dr. K. Soteriadou, Laboratory of Molecular Parasitology, Hellenic Pasteur Institute, for discussion, input, and for kindly providing the parasites. IDK received financial support from the Development Grants for Research Institutions-KRIPIS from the Greek General Secretariat for Research and Technology (Ministry of Education). 
Citation: Kyriazis ID, Smirlis D, Papadaki A, Koutsoni O, Aligiannis N, et al. (2017) Leishmanicidal Activity of Oleuropein: Leishmania donovani Promastigote Cell Death through a Possibly ROS-Independent Mechanism. J Pharmacogn Nat Prod 3: 141. doi: $10.4172 / 2472-0992.1000141$

Page 7 of 7

\section{References}

1. Rocha LG, Almeida JR, Macedo RO, Barbosa-Filho JM (2005) A review of natural products with antileishmanial activity. Phytomedicine 12: 514-535.

2. Omar SH (2010) Oleuropein in olive and its pharmacological effects. Scientia pharmaceutica 78: 133-154.

3. Samara P, Christoforidou N, Lemus C, Argyropoulou A, Ioannou K, et al (2017) New semi-synthetic analogs of oleuropein show improved anticancer activity in vitro and in vivo. Eur J Med Chem 137: 11-29.

4. Kyriazis JD, Aligiannis N, Polychronopoulos P, Skaltsounis AL, Dotsika E (2013) Leishmanicidal activity assessment of olive tree extracts. Phytomed 20: 275-281.

5. Kroemer G, Galluzzi L, Vandenabeele P, Abrams J, Alnemri ES, et al. (2009) Classification of cell death: recommendations of the Nomenclature Committee on Cell Death 2009. Cell Death Differ 16: 3.

6. Han J, Talorete TP, Yamada P, Isoda H (2009) Anti-proliferative and apoptotic effects of oleuropein and hydroxytyrosol on human breast cancer MCF-7 cells. Cytotechnology 59: 45-53.

7. Abe R, Beckett J, Nixon A, Rochier A, Yamashita N, et al. (2011) Olive oil polyphenol oleuropein inhibits smooth muscle cell proliferation. Eur J Vasc Endovasc Surg 41: 814-820.

8. Cárdeno A, Sánchez-Hidalgo M, Rosillo MA, de la Lastra CA (2013) Oleuropein, a secoiridoid derived from olive tree, inhibits the proliferation of human colorectal cancer cell through downregulation of HIF-1a. Nutr Cancer 65: 147-156.

9. Smirlis D, Soteriadou K (2011) Trypanosomatid apoptosis:'Apoptosis' without the canonical regulators. Virulence 2: 253-256.

10. Smirlis D, Duszenko M, Ruiz A, Scoulica E, Bastien P, et al. (2010) Targeting essential pathways in trypanosomatids gives insights into protozoan mechanisms of cell death. Parasites \& Vectors [Internet]. Springer Nature 3: 107.

11. WHO (2010) Working to overcome the global impact of neglected tropical diseases.

12. Freitas-Junior LH, Chatelain E, Kim HA, Siqueira-Neto JL (2012) Visceral leishmaniasis treatment: what do we have, what do we need and how to deliver it?. Int J Parasitol Drugs Drug Resist 2: 11-9.

13. Singh N, Mishra BB, Bajpai S, Singh RK, Tiwari VK (2014) Natural product based leads to fight against leishmaniasis. Bioorganic Med Chem 22: $18-45$.

14. van Zandbergen G, Bollinger A, Wenzel A, Kamhawi S, Voll R, Klinger $\mathrm{M}$, et al. (2006) Leishmania disease development depends on the presence of apoptotic promastigotes in the virulent inoculum. Proc Natl Acad Sci U.S.A 103: 13837-13842.

15. Messmer UK, Pfeilschifter J (2000) New insights into the mechanism for clearance of apoptotic cells. Bioessays 22: 878-881.

16. Moreira ME, Barcinski MA (2004) Apoptotic cell and phagocyte interplay: recognition and consequences in different cell systems. An Acad Bras Cienc 76: 93-115.

17. Kyriazis ID, Koutsoni OS, Aligiannis N, Karampetsou K, Skaltsounis AL, et al. (2016) The leishmanicidal activity of oleuropein is selectively regulated through inflammation-and oxidative stress-related genes. Parasit Vectors 9: 441.

18. Eruslanov E, Kusmartsev S (2010) Identification of ROS using oxidized DCFDA and flow-cytometry. Advanced protocols in oxidative stress II : 57-72.

19. Dutta A, Bandyopadhyay S, Mandal C, Chatterjee M (2007) Aloe vera leaf exudate induces a caspase-independent cell death in Leishmania donovani promastigotes. J Med Microbiol 56: 629-636.

20. Latha ST, Rajendran NN (2013) Apoptosis Effect Of Isolated Phenolic Secoiridoid Glucoside From Aerial Parts Of Gomphrena Globosa In A-431cell Lines. World J Pharm and Pharmaceut Scienc. 2(5): 3829-3839.

21. Voronov E, Dotan S, Gayvoronsky L, White RM, Cohen I, et al. (2010) IL-1-induced inflammation promotes development of leishmaniasis in susceptible BALB/c mice. Int Immunol 22: 245-257.

22. Dutta A, Ghoshal A, Mandal D, Mondal NB, Banerjee S, et al. (2007) Racemoside A, an anti-leishmanial, water-soluble, natural steroidal saponin, induces programmed cell death in Leishmania donovani. J Med Microbiol 56: 1196-1204.

23. Sen R, Bandyopadhyay S, Dutta A, Mandal G, Ganguly S, et al. (2007) Artemisinin triggers induction of cell-cycle arrest and apoptosis in Leishmania donovani promastigotes. J Med Microbiol 56: 1213-1218.

24. Georgopoulou K, Smirlis D, Bisti S, Xingi E, Skaltsounis L, et al. (2007) In vitro activity of 10-deacetylbaccatin III against Leishmania donovani promastigotes and intracellular amastigotes. Planta medica 73 : 1081-1088.

25. Mittra B, Saha A, Chowdhury AR, Pal C, Mandal S, et al. (2000) Luteolin, an abundant dietary component is a potent anti-leishmanial agent that acts by inducing topoisomerase II-mediated kinetoplast DNA cleavage leading to apoptosis. Mol Med. 6: 527-541.

26. Van Engeland M, Nieland LJ, Ramaekers FC, Schutte B, Reutelingsperger CP, et al. (1998) Annexin V-affinity assay: a review on an apoptosis detection system based on phosphatidylserine exposure. cytometry 31 : $1-9$.

27. Weingärtner A, Kemmer G, Müller FD, Zampieri RA, dos Santos MG, et al. (2012) Leishmania promastigotes lack phosphatidylserine but bind annexin $\mathrm{V}$ upon permeabilization or miltefosine treatment. PloS one 7: e42070.

28. Dutta A, Sarkar D, Gurib-Fakim A, Mandal C, Chatterjee M (2008) In vitro and in vivo activity of Aloe vera leaf exudate in experimental visceral leishmaniasis. J Parasitol Res 102: 1235-1242.

29. Irigoín F, Inada NM, Fernandes MP, Piacenza L, Gadelha FR, et al. (2009) Mitochondrial calcium overload triggers complement-dependent superoxide-mediated programmed cell death in Trypanosoma cruzi. Biochemical J 418: 595-604.

30. Das R, Roy A, Dutta N, Majumder HK (2008) Reactive oxygen species and imbalance of calcium homeostasis contributes to curcumin induced programmed cell death in Leishmania donovani. Apoptosis 13: 867-882.

31. Sudhandiran G, Shaha C (2003) Antimonial-induced increase in intracellular $\mathrm{Ca}^{2+}$ through non-selective cation channels in the host and the parasite is responsible for apoptosis of intracellular Leishmania donovani amastigotes. J Biol Chem 278: 25120-25132. 